\title{
Exploratory/Developmental Grant for Diagnostic Cancer Imaging
}

National Cancer Institute

\section{Source}

National Cancer Institute. Exploratory/Developmental Grant for Diagnostic Cancer

Imaging. NCl Thesaurus. Code C19262.

The Diagnostic Imaging Program (DIP), of the Division of Cancer Treatment and Diagnosis (DCTD) solicits exploratory/developmental (R21) grants that articulate highly innovative research concepts in diagnostic cancer imaging to provide investigators with the initial resources required to accomplish feasibility and pilot testing of innovative ideas. The R21 mechanism will provide investigators at all career levels with a defined level of funding adequate for the initial feasibility testing of high risk/high impact concepts and, if the concepts are viable, for the generation of experimental preliminary data. This would render the investigators competitive for funding through the research project grant (R01) and/or First Independent Research Support and T ransition (FIRST) (R29) award mechanisms, thus potentially leading to the establishment of new research programs in areas that might have previously remained unexplored. 